\title{
Single cell genotyping of matched bone marrow and peripheral blood cells in treatment naive and AZA-treated MDS and CMML
}

Running title: Single cell genotyping in MDS/CMML

Annatina Schnegg-Kaufmann 1, 2, \#, Julie A. I. Thoms ${ }^{1, \#, ~ G o l a m ~ S a r o w e r ~ B h u y a n ~}{ }^{3}$, Henry R. Hampton ${ }^{1}$, Lachlin Vaughan ${ }^{3,4,5}$, Kayleigh Rutherford ${ }^{6}$, Purvi M. Kakadia ${ }^{7}$, Emma M. V. Johansson $^{8}$, Tim Failes ${ }^{9}$, Greg M. Arndt ${ }^{9}, 10,11$, Jason Koval ${ }^{12}$, Robert Lindeman ${ }^{13}$, Pauline Warburton ${ }^{14}$, Alba Rodriguez-Meira ${ }^{15}$, Adam J. Mead ${ }^{15}$, Ashwin Unnikrishnan ${ }^{3}$, Stefan K. Bohlander $^{7}$, Elli Papaemmanuil ${ }^{6}$, Omid Faridani 1, 16, 16, *, Christopher J. Jolly ${ }^{1,}$ *, Fabio Zanini $3,16,17,{ }^{*},+$, and John E. Pimanda $1,3,11, *,+$

${ }^{1}$ School of Medical Sciences, Lowy Cancer Research Centre, UNSW Sydney, Sydney, NSW, Australia

${ }^{2}$ Department of Haematology and Central Haematology Laboratory, Inselspital Bern, University Hospital and University of Bern, Bern, Switzerland.

${ }^{3}$ Prince of Wales Clinical School, Lowy Cancer Research Centre, UNSW Sydney, Sydney, NSW, Australia

${ }^{4}$ Institute of Clinical Pathology and Medical Research, Westmead Hospital, Sydney, NSW, Australia

${ }^{5}$ Haematology Department, Westmead Hospital, Sydney, NSW, Australia

${ }^{6}$ Computational Oncology Service, Department of Epidemiology \& Biostatistics, Center for Computational Oncology, Memorial Sloan Kettering Cancer Center, New York, NY, USA

${ }^{7}$ Leukaemia and Blood Cancer Research Unit, Department of Molecular Medicine and Pathology, University of Auckland, Auckland, New Zealand

${ }^{8}$ Flow Cytometry Facility, Mark Wainwright Analytical Centre, UNSW Sydney, Sydney, NSW, Australia

${ }^{9}$ Children's Cancer Institute, Lowy Cancer Research Centre, UNSW Sydney, Sydney, NSW, Australia

${ }^{10}$ Australian Cancer Research Foundation (ACRF) Drug Discovery Centre for Childhood Cancer, Children's Cancer Institute, Lowy Cancer Research Centre, UNSW Sydney, Sydney, NS W, Australia

${ }^{11}$ School of Women's and Children's Health, UNSW Sydney, Sydney, NSW, Australia

${ }^{12}$ Ramaciotti Centre for Genomics, UNSW Sydney, Sydney, NSW, Australia

${ }^{13}$ Department of Haematology, Prince of Wales Hospital, Sydney, NSW, Australia

${ }^{14}$ Department of Haematology, Wollongong Hospital, Wollongong, NSW, Australia

15 Haematopoietic Stem Cell Biology Laboratory, Weatherall Institute of Molecular Medicine, University of Oxford, Oxford, UK

${ }^{16}$ Garvan-Weizmann Centre for Cellular Genomics, Sydney, NSW, Australia

${ }^{17}$ UNSW Cellular Genomics Futures Institute, Sydney, NSW, Australia

\section{${ }^{\#}$ Equal contributions, + Senior equal contribution}

*Corresponding authors: John Pimanda - jpimanda@unsw.edu.au, Fabio Zanini fabio.zanini@unsw.edu.au, Chris Jolly - c.jolly@unsw.edu.au, Omid Faridani o.faridani@unsw.edu.au. Level 2, Lowy Cancer Research Centre, UNSW Sydney, NSW 2052, Australia, P: +612 93852527 F: +612 93851510

Word count (abstract, $\max 200$ ): 191, Word count (text, $\max 1200$ ): 1291, Figure count: 2 main, 5 supplemental, Table count: 8 supplemental, Reference count: 25

Keywords: myelodysplastic syndromes, chronic myelomonocytic leukaemia, single cell genotyping, azacytidine therapy 


\section{Key points}

* Highly mutated HSPCs contribute significantly to circulating blood cells in MDS and CMML, prior to and following AZA treatment.

* The mutational burden in matched bone marrow and peripheral blood cells in MDS and CMML was similar throughout myelopoiesis. 


\begin{abstract}
Progressively acquired somatic mutations in hematopoietic stem cells are central to pathogenesis in myelodysplastic syndromes (MDS) and chronic myelomonocytic leukemia (CMML). They can lead to proliferative advantages, impaired differentiation and progressive cytopenias. MDS or CMML patients with high-risk disease are treated with hypomethylating agents including 5-azacytidine (AZA). Clinical improvement does not require eradication of mutated cells and may be related to improved differentiation capacity of mutated hematopoietic stem and progenitor cells (HSPCs). However, the contribution of mutated HSPCs to steadystate hematopoiesis in MDS and CMML is unclear. To address this, we characterised the somatic mutations of individual stem, progenitor (common myeloid progenitor, granulocyte monocyte progenitor, megakaryocyte erythroid progenitor), and matched circulating (monocyte, neutrophil, naïve B cell) haematopoietic cells in treatment naïve and AZA-treated MDS and CMML via high-throughput single cell genotyping. The mutational burden was similar across multiple hematopoietic cell types, and even the most mutated stem and progenitor clones maintained their capacity to differentiate to mature myeloid and, in some cases, lymphoid cell types in vivo. Our data show that even highly mutated HSPCs contribute significantly to circulating blood cells in MDS and CMML, prior to and following AZA treatment.
\end{abstract}




\section{INTRODUCTION}

Somatic mutations in hematopoietic stem cells (HSCs) are a central pathogenic event in myelodysplastic syndromes (MDS) and chronic myelomonocytic leukemia (CMML) where they induce proliferative advantages and impaired differentiation with subsequent cytopenias in peripheral blood $(\mathrm{PB})^{1-6}$. Patients with high-risk disease who are ineligible for transplantation are treated with hypomethylating agents, usually 5-azacytidine (AZA). AZA treatment can improve PB counts and delay progression to AML in some patients ${ }^{7-9}$. However, AZA response does not require eradication of mutated HSCs. We and others have previously described cohorts with haematological response to AZA despite persistently high variant allele fractions in bone marrow $(\mathrm{BM}){ }^{10,11}$. Colonies derived from in vitro assays of stem cell function following AZA treatment showed decreased mutational complexity, suggesting a shift in haematopoiesis from clones with high to low mutational burden in response to treatment ${ }^{11}$. However, in vitro colony-forming capacity might not correlate with in vivo hematopoietic potential, and the relative contribution of high vs low/unmutated HSCs to circulating blood cell types in vivo remains unknown. Single cell genotyping techniques can be used to resolve combinations of mutations in ex vivo cells ${ }^{1,12-15}$. However, to our knowledge such techniques have not been applied to resolve the relative contributions of stem and progenitor cells with multiple mutations to circulating mature blood cells in MDS/CMML. Here we use a combination of index sorting and single cell genotyping to characterise the haplotype composition of individual stem (HSC/multipotent progenitors (MPP), MDS stem cells (MDS$\mathrm{SC}$ ), progenitor (common myeloid progenitor (CMP), granulocyte monocyte progenitor (GMP), megakaryocyte erythroid progenitor (MEP)), and high-turnover circulating cells (monocyte, neutrophil, naïve B cell (nBC)) in treatment naïve and AZA-treated MDS and CMML. 


\section{METHODS}

BM and PB samples were collected with patient consent and institutional ethics approval. BM was enriched for CD34+ cells and target populations single-cell index sorted into 384-well plates (Figure S1). Target mutations were amplified in single cells by modifying TARGETSeq ${ }^{15}$ followed by barcoding and Illumina sequencing (Figure S2; Supplemental Material). Capture sequencing was performed using a targeted panel for myeloid driver mutations (Supplementary Material). Raw data is available at SRA (accession: PRJNA798507) and https://flowrepository.org/id/FR-FCM-Z4PR. Mutational calling was performed via pairwise sequence alignments using SeqAn ${ }^{16}$ and seqanpy (https://github.com/iosonofabio/seqanpy). Analysis code is available at https://github.com/julie-thoms/MDS amplicons. 


\section{RESULTS and DISCUSSION}

We analysed matched stem and progenitor cells from BM and high-turnover differentiated cells from PB in three patients (Figure 1A, B, Table S1). Variant allele fractions (VAFs) were determined by capture sequencing in bulk samples from each cell type. The distribution of VAFs were similar across BM and PB cell types in all patients with the notable exception that nBCs in patient H198304 were predominantly wild-type (wt) (Figure 1C). We confirmed this exception using an orthogonal approach (Figure S3). We then modified TARGET-Seq to determine VAFs of these known mutations in single cells (Figures 1D, S4). Allele fractions were highly correlated between bulk- and single-cell assessments (Figure S5; Pearson $\mathrm{r}=$ $0.8989)$.

Stem cells with multiple mutations (Figure 2A) could be subject to negative clonal selection with a bias towards less mutated stem and progenitor cells contributing to circulating differentiated cells, or neutral selection with similar contributions irrespective of mutational burden (Figure 2B). To resolve this, we first classified BM cells from each of the three patients into healthy HSC/MPP, MDS-SC, CMP, GMP, or MEP using indexed fluorescence activated cell sorting (FACS) and assessed the presence/absence of known variants in each of these BM cell types and in matched PB neutrophils, monocytes and nBCs (Figure 2C-E).

In patient H198302 (CMML; AZA therapy 10 years; complete responder $\left(\mathrm{IWG}^{17}\right.$ ); Table S1), we tracked four mutations (Figure 2C; SRSF2, CUX1 and biallelic TET2), with acquisition order inferred from VAF at diagnosis ${ }^{11}$. Most stem cells carried two or three of the tracked mutations and we did not detect any wt HSC/MPP. Cells across the progenitor compartment were similar and mostly highly mutated - a pattern that was maintained 
particularly in differentiated monocytes and neutrophils with a substantial proportion of the circulating cells derived from highly mutated progenitors.

In patient H198303 (CMML; AZA therapy 10 years; complete responder $\left(\mathrm{IWG}^{17}\right.$ ); Table S1), we tracked two mutations detected at diagnosis ${ }^{11}$ (Figure 2D; biallelic TET2). In the stem compartment, cells carrying biallelic TET2 mutations were frequent, but numerous cells carried either a single mutant allele or were wt. Progenitors were relatively homogenous, although mutational burden differed slightly from the stem compartment. The haplotype distribution in differentiated cells was again similar to progenitor cells, with the exception that we did not detect any wt neutrophils.

In patient H198304 (MDS-EB1; no HMA therapy), we tracked four mutations (Figure 2E; SRSF2, RUNX1 and biallelic TET2). Most stem cells carried two or three mutations, a few healthy HSC/MPPs had no mutations detected, and around 20\% of MDS-SC carried an additional mutated $R U N X 1$ allele. In the progenitor compartment all cell types were similar; most cells carried three mutations. In the differentiated compartment, myeloid populations contained cells with one, two, three, or four mutations, with a higher proportion of cells with all four alleles mutated than progenitors, suggesting that highly mutated stem cells can produce differentiated myeloid cells, and that cells carrying the additional $R U N X 1$ mutation are advantaged in this regard compared to cells with TET2 and SRSF2 mutations alone. RUNX1_p.A187T lies within the DNA- and protein-binding runt homology domain and may alter transcriptional activity. Conditional $R U N X 1$ knockout leads to myeloid proliferation ${ }^{18}$; the patient's mutation may similarly favour myeloid expansion or differentiation. Consistent with bulk analysis, very few mutant cells were detected in $\mathrm{nBCs}$, suggesting that the small wt 
HSC/MPP population is the predominant origin of $\mathrm{nBCs}$ in this individual. Analysis of additional lymphoid populations in PB from this patient revealed that naïve $\mathrm{T}$, but not NK, cells were also predominantly wt (Figure S3C-D), suggesting specific impairment of B- and Tlineage maturation in the mutated cells.

All patients had clones with biallelic TET2 mutations, with or without additional oncogenic mutations, and shared the following features: (a) Very few, if any, sampled HSCs (with or without aberrant $\mathrm{CD}_{4} 5 \mathrm{RA}^{+}$or $\mathrm{CD} 123^{+}$or $\mathrm{IL}_{\mathrm{RAP}}{ }^{+}$) were wild-type, (b) A high proportion of sampled HSCs and myeloid progenitors in the bone marrow had two or more mutations, (c) Few, if any of the sampled circulating neutrophils or monocytes were wild-type, (d) The mutation profiles of PB neutrophils and monocytes (very high-turnover cells) mirrored those that were present in corresponding myeloid progenitors in the bone marrow. Attrition of highly mutated cells during myeloid maturation was not observed in any of the three patients, irrespective of HMA therapy, suggesting that in vivo, highly mutated stem and progenitor cells retain the capacity to differentiate. However, there were individual differences. In particular, in patient $\mathrm{H} 198304$ the rarity of mutated nBC compared to mutant $\mathrm{NK}$ and myeloid cells might be attributed to the order of mutation acquisition ${ }^{19-22}$, the presence of an additional undetected somatic mutation in mutant cells that specifically impacts B cells, or individual differences in the bone marrow microenvironment ${ }^{23,24}$.

In summary, we characterised the mutational profile of thousands of individual stem, progenitor, and differentiated cells from three MDS/CMML patients and found that in vivo, highly mutated stem cells contribute to the circulating population prior to and following AZA treatment and were preferentially poised towards specific differentiation trajectories. These 
findings are particularly relevant in an era of combining cytotoxic therapies designed to eliminate mutant cells with hypomethylating agents ${ }^{25}$. Our findings emphasise that therapeutic principles and endpoints that apply in high-blast AML (clonal eradication and minimal residual disease monitoring for relapse) may not be appropriate when treating high-risk MDS/CMML where clinical response does not require eradication of mutant clones; indeed, eradication may be detrimental. Further studies will help determine the prevalence of this phenomenon among cohorts of MDS and CMML patients with different mutation profiles and whether it correlates with clinical outcomes. 


\section{ACKNOWLEDGEMENTS}

We thank study participants for their generosity in providing samples, and Swapna Joshi and Ameline Lim for assistance in processing PB and BM. AS-K was supported by a Swiss national research foundation early postdoc mobility grant (P2LAP3_181273). SKB and PMK are supported by Leukaemia and Blood Cancer New Zealand and the family of Marijana Kumerich. JEP is supported by project grants from the National Health and Medical Research Council of Australia (APP1042934, APP1102589, APP1008515), a translational program grant from the Leukemia Lymphoma Society (LLS)-Snowdome Foundation-Leukaemia Foundation, project funds from the Translational Cancer Research Network - a Translational Cancer Research Centre funded by the Cancer Institute NSW, Anthony Rothe Memorial Trust, and philanthropic funding from Christina's Light. 


\section{AUTHORSHIP CONTRIBUTIONS}

AS-K, JAIT, RL, PW, AR-M, AJM, AU, OF, CJJ, FZ, JEP designed research.

AS-K, JAIT, GSB, HRH, LV, PMK, EMVJ, TF, GMA, JK, OF, CJJ performed research.

AS-K, JAIT, KR, PMK, SKB, EP, CJJ, FZ, JEP analyzed and interpreted data.

AS-K, JAIT, CJJ, FZ, JEP wrote the manuscript. 


\section{DISCLOSURES of CONFLICTS of INTEREST}

The authors declare no conflicts of interest. 


\section{REFERENCES}

1. Chen J, Kao YR, Sun D, et al. Myelodysplastic syndrome progression to acute myeloid leukemia at the stem cell level. Nat Med. 2019;25(1):103-110.

2. Papaemmanuil E, Gerstung M, Malcovati L, et al. Clinical and biological implications of driver mutations in myelodysplastic syndromes. Blood. 2013;122(22):3616-3627; quiz 3699.

3. Carr RM, Patnaik MM. Genetic and epigenetic factors interacting with clonal hematopoiesis resulting in chronic myelomonocytic leukemia. Curr Opin Hematol. 2020;27(1):2-10.

4. Woll PS, Kjallquist U, Chowdhury O, et al. Myelodysplastic syndromes are propagated by rare and distinct human cancer stem cells in vivo. Cancer Cell. 2014;25(6):794-808.

5. Bejar R, Stevenson K, Abdel-Wahab O, et al. Clinical effect of point mutations in myelodysplastic syndromes. N Engl J Med. 2011;364(26):2496-2506.

6. Haferlach T, Nagata Y, Grossmann V, et al. Landscape of genetic lesions in 944 patients with myelodysplastic syndromes. Leukemia. 2014;28(2):241-247.

7. Fenaux P, Mufti GJ, Hellstrom-Lindberg E, et al. Efficacy of azacitidine compared with that of conventional care regimens in the treatment of higher-risk myelodysplastic syndromes: a randomised, open-label, phase III study. Lancet Oncol. 2009;10(3):223-232.

8. Silverman LR, Demakos EP, Peterson BL, et al. Randomized controlled trial of azacitidine in patients with the myelodysplastic syndrome: a study of the cancer and leukemia group B. J Clin Oncol. 2002;20(10):2429-2440.

9. Bejar R, Lord A, Stevenson K, et al. TET2 mutations predict response to hypomethylating agents in myelodysplastic syndrome patients. Blood. 2014;124(17):27052712.

10. Merlevede J, Droin N, Qin T, et al. Mutation allele burden remains unchanged in chronic myelomonocytic leukaemia responding to hypomethylating agents. Nat Commun. 2016;7:10767.

11. Unnikrishnan A, Papaemmanuil E, Beck D, et al. Integrative Genomics Identifies the Molecular Basis of Resistance to Azacitidine Therapy in Myelodysplastic Syndromes. Cell Rep. 2017;20(3):572-585.

12. Rodriguez-Meira A, O'Sullivan J, Rahman H, Mead AJ. TARGET-Seq: A Protocol for High-Sensitivity Single-Cell Mutational Analysis and Parallel RNA Sequencing. STAR Protoc. 2020;1(3):100125.

13. Miles LA, Bowman RL, Merlinsky TR, et al. Single-cell mutation analysis of clonal evolution in myeloid malignancies. Nature. 2020;587(7834):477-482.

14. Morita K, Wang F, Jahn K, et al. Clonal evolution of acute myeloid leukemia revealed by high-throughput single-cell genomics. Nat Commun. 2020;11(1):5327.

15. Rodriguez-Meira A, Buck G, Clark SA, et al. Unravelling Intratumoral Heterogeneity through High-Sensitivity Single-Cell Mutational Analysis and Parallel RNA Sequencing. Mol Cell. 2019;73(6):1292-1305 e1298.

16. Reinert K, Dadi TH, Ehrhardt M, et al. The SeqAn C++ template library for efficient sequence analysis: A resource for programmers. J Biotechnol. 2017;261:157-168.

17. Cheson BD, Greenberg PL, Bennett JM, et al. Clinical application and proposal for modification of the International Working Group (IWG) response criteria in myelodysplasia. Blood. 2006;108(2):419-425.

18. Growney JD, Shigematsu H, Li Z, et al. Loss of Runx1 perturbs adult hematopoiesis and is associated with a myeloproliferative phenotype. Blood. 2005;106(2):494-504. 
19. Meggendorfer M, Roller A, Haferlach T, et al. SRSF2 mutations in 275 cases with chronic myelomonocytic leukemia (CMML). Blood. 2012;120(15):3080-3088.

20. Thol F, Kade S, Schlarmann C, et al. Frequency and prognostic impact of mutations in SRSF2, U2AF1, and ZRSR2 in patients with myelodysplastic syndromes. Blood. 2012;119(15):3578-3584.

21. Wu SJ, Kuo YY, Hou HA, et al. The clinical implication of SRSF2 mutation in patients with myelodysplastic syndrome and its stability during disease evolution. Blood. 2012;120(15):3106-3111.

22. Inoue D, Bradley RK, Abdel-Wahab O. Spliceosomal gene mutations in myelodysplasia: molecular links to clonal abnormalities of hematopoiesis. Genes Dev. 2016;30(9):989-1001.

23. Bhagat TD, Chen S, Bartenstein M, et al. Epigenetically Aberrant Stroma in MDS Propagates Disease via Wnt/beta-Catenin Activation. Cancer Res. 2017;77(18):4846-4857.

24. Pronk E, Raaijmakers M. The mesenchymal niche in MDS. Blood. 2019;133(10):10311038.

25. DiNardo CD, Jonas BA, Pullarkat V, et al. Azacitidine and Venetoclax in Previously Untreated Acute Myeloid Leukemia. N Engl J Med. 2020;383(7):617-629. 


\section{FIGURE LEGENDS}

Figure 1 - Mutational burden in bone marrow progenitors and differentiated peripheral blood cells

(A) Diagnosis and tracked mutations in the three patients included in this study. (B) Left: Blood differentiation hierarchy in MDS/CMML showing stem (healthy stem cells [HSC/MPP] and MDS stem cells [MDS-SC]), progenitor (CMP, GMP, MEP, CLP), and differentiated mature cells. Cell types coloured white were not characterised in this study. Right: Schematic showing collection and cell sorting strategies for peripheral blood (PB) and bone marrow (BM). PB was flow sorted into neutrophils (Neut: $\mathrm{SSC}^{\text {hi }}, \mathrm{CD}^{+} 5^{+}, \mathrm{IgD}^{-}, \mathrm{CD}^{+} 6^{+}, \mathrm{CD} 6 \mathrm{~b}^{+}$), monocytes (Mono: $\left.\mathrm{SSC}^{\mathrm{lo}}, \mathrm{CD}^{4} 5^{+}, \mathrm{IgD}^{-}, \mathrm{CD} 16^{+}\right)$, and naïve $\mathrm{B}$ cells $\left(\mathrm{nBC}: \mathrm{SSC}^{\mathrm{lo}}, \mathrm{CD}^{4} 5^{+}, \mathrm{IgD}^{+}, \mathrm{CD} 27^{-}\right)$. Bone marrow mononuclear cells (BM-MNCs) were isolated on Ficoll and used directly for bulk capture sequencing. MACS-enriched CD34+ cells (BM-CD34+) were dropped into 384 wells plates for amplicon sequencing, with indexing for CD38, CD123, CD45RA, CD90, and IL1RAP and post-hoc assignment of cell type (HSC/MPP: LIN ${ }^{-}, \mathrm{CD}^{+} 4^{+}, \mathrm{CD}^{10}{ }^{\text {lo }}, \mathrm{CD} 45 \mathrm{RA}^{-}$, CD123-, IL1RAP ${ }^{-}$; MDS-SC: $\mathrm{LIN}^{-}, \mathrm{CD}^{+} 4^{+}, \mathrm{CD}^{\text {lo }}{ }^{\circ}, \mathrm{CD}_{45 \mathrm{RA}^{+}}$or $\mathrm{CD} 123^{+}$or $\mathrm{IL}_{\mathrm{RAP}}{ }^{+}$;

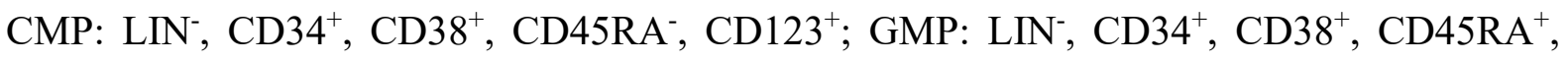
$\mathrm{CD}^{2} 3^{+}$; MEP: $\left.\mathrm{LIN}^{-}, \mathrm{CD} 34^{+}, \mathrm{CD} 38^{+}, \mathrm{CD} 4 \mathrm{RA}^{-}, \mathrm{CD}^{2} 3^{-}\right)$(C) Variant allele fraction (VAFs) determined by capture sequencing in bulk BM and PB cell types. (D) VAFs determined by amplicon sequencing in single cells. Each allele was analysed individually. Upper bar graph indicates the number of cells analysed for each mutation in each cell type. Lower graph shows average VAF (i.e., the proportion of reads containing the known mutation) across all cells with at least 10 reads mapping to that amplicon. Bars show standard error of the mean (sem). 
Figure 2 - Fitness of highly mutated MDS and CMML stem cells to produce terminal blood types

(A) Schematic showing hypothetical clonal evolution in MDS/CMML with sequential acquisition of mutations in four alleles (allele $\mathrm{A}$, allele $\mathrm{B}$, allele $\mathrm{C}$, allele $\mathrm{D}$; note that in some cases both alleles of a single gene may independently acquire mutations e.g., in the case of TET2). The combined genotype (haplotype) of each resulting cell population is indicated, with lower case letters indicating the presence of a mutated allele. (B) Models of terminal blood production in MDS/CMML. In the neutral selection model (left) stem cells with multiple mutations retain capacity to produce terminal blood types, although there may be a reduction in absolute cell number or functionality. In the negative clonal selection model (right), cells harbouring multiple mutant alleles are abundant in the stem cell compartment but have reduced differentiation capacity resulting in a higher frequency of wild-type cells in the circulating population. (C-E) Single cell haplotypes in three patients. Heatmaps (left panels) show the observed frequency of all combinations of mutations, while pie charts (right panels) show the proportions of cells across the haematopoietic hierarchy carrying zero, one, two, three, or four mutations in the specified alleles. (C) Patient H198302. Alleles shown are TET2_p.Q686* (b), TET2_p.L1065fs*1 (a), SRSF2_p.P95H (s), CUX1_p.Q1276* (c). (D) Patient H198303. Alleles shown are TET2_p.A1224fs*2 (b), TET2_p.K1090fs*15 (a). (E) Patient H198304. Alleles shown are SRSF2_p.P95R (s), TET2_p.G1218fs*8 (a), TET2_p.Y1337*, (b) RUNX1_p.A187T $(r)$. 
A
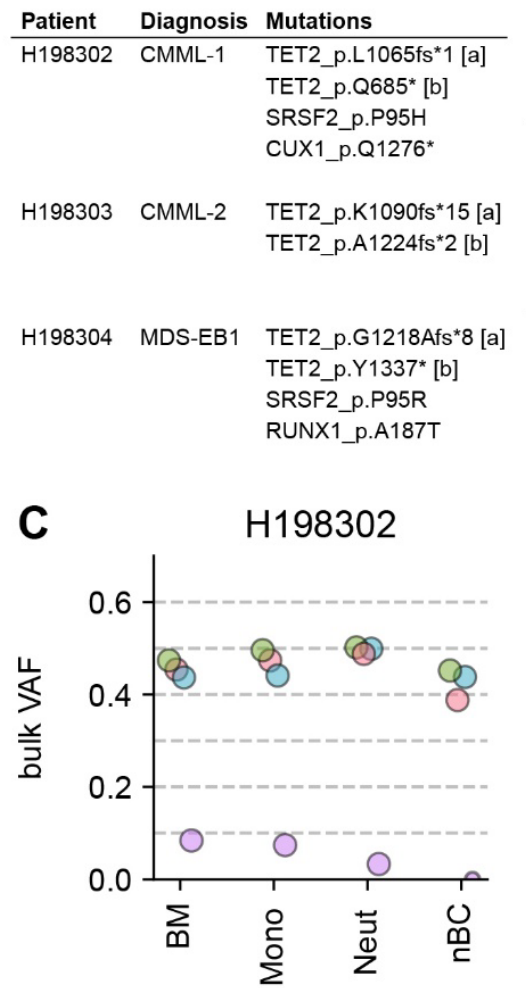
TET2[a]
O TET2[b]
SRSF2
CUX1

B
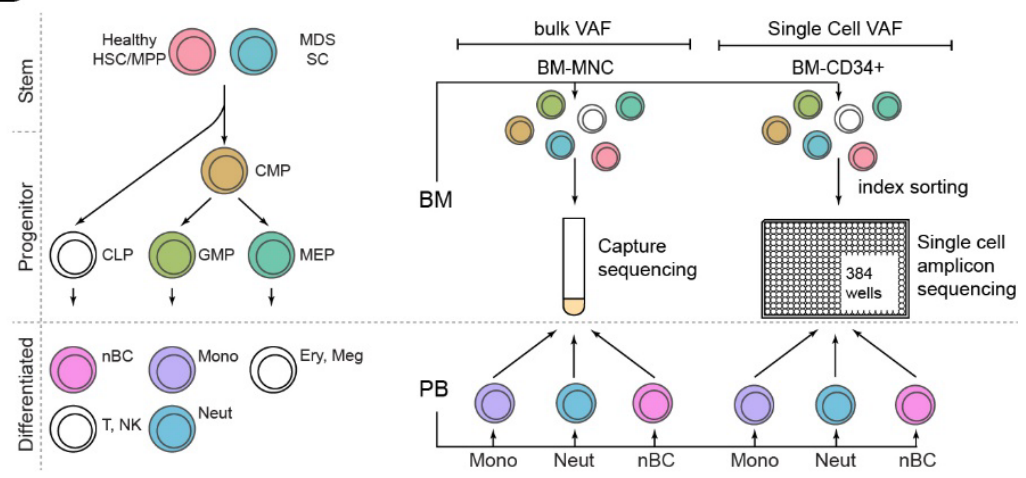

H198303
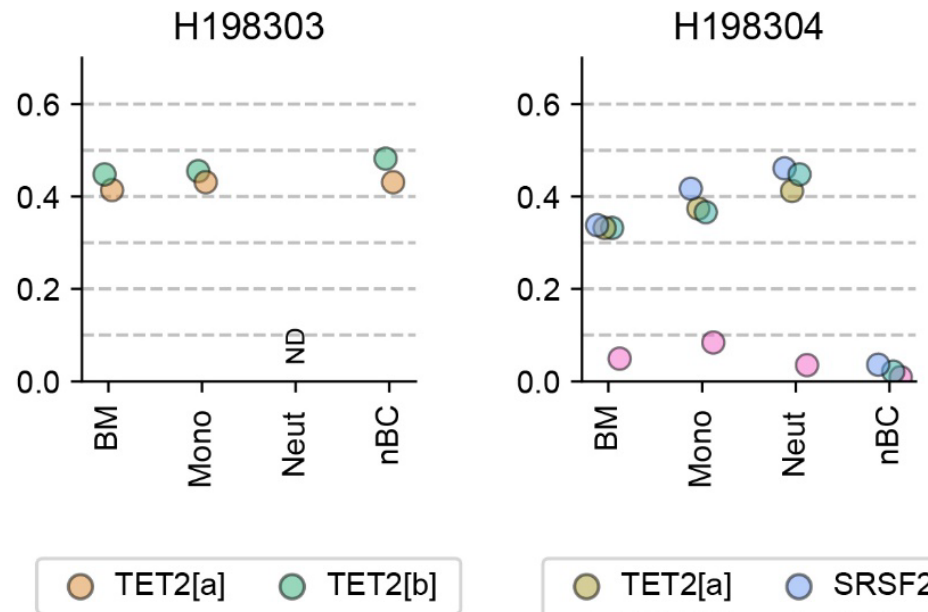

TET2[a] $\bigcirc$ SRSF2

$\bigcirc$ TET2[b] $\bigcirc$ RUNX1
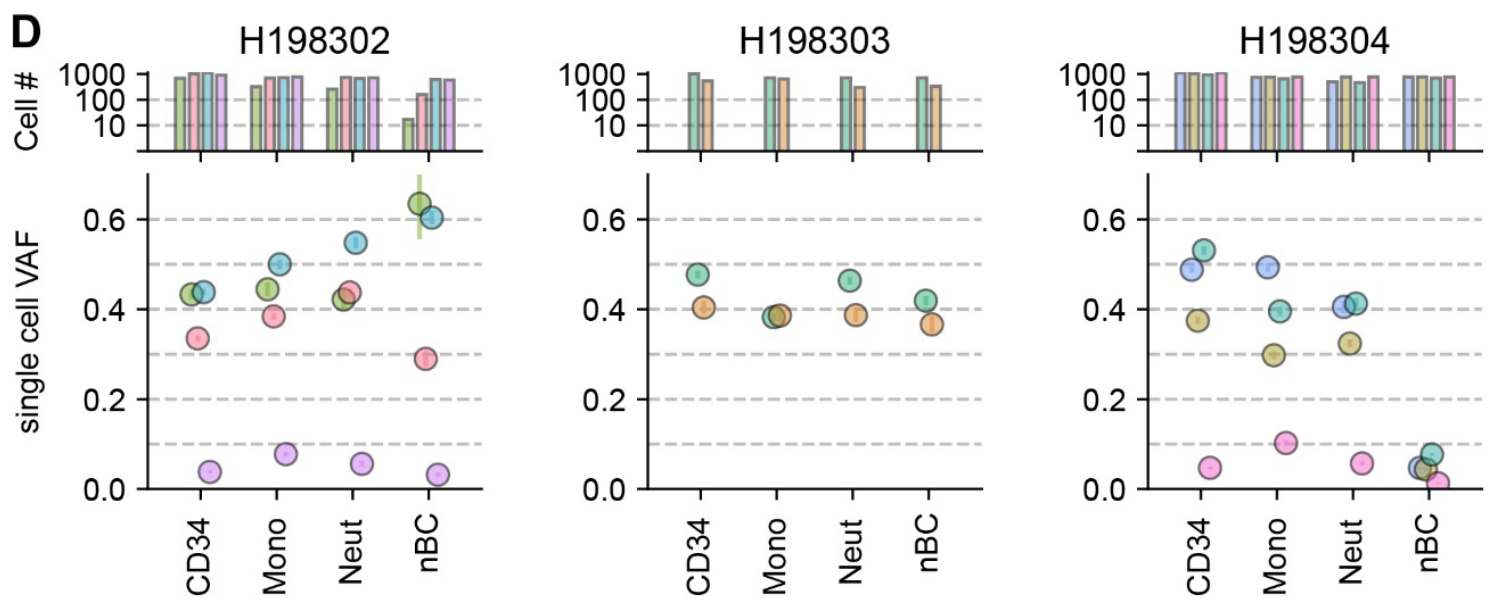
○ TET2[a]
SRSF2
O TET2[b]
CUX1

\begin{tabular}{|c|c|}
\hline$)^{T}$ & \\
\hline $\mathrm{TE}$ & \\
\hline
\end{tabular}

Figure 1 
bioRxiv preprint doi: https://doi.org/10.1101/2022.01.20.476890; this version posted January 22, 2022. The copyright holder for this preprint (which was not certified by peer review) is the author/funder. All rights reserved. No reuse allowed without permission.

A

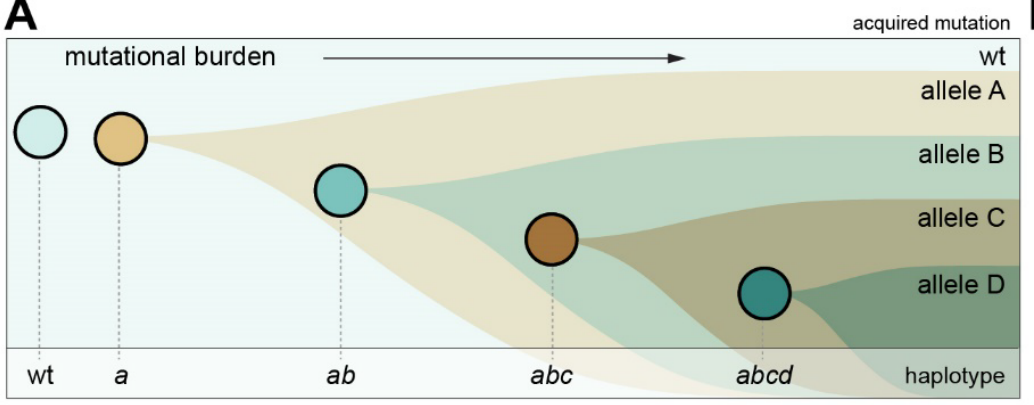

Clonal evolution (stem cell compartment)

C
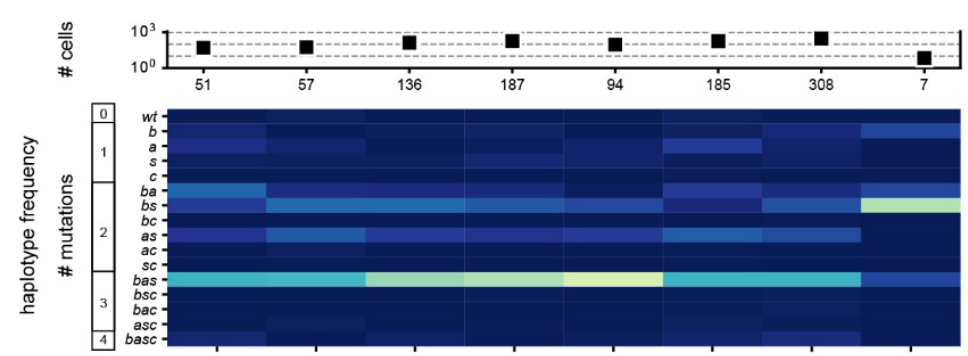

(b) TET2_P.Q685*

(a) TET2_p.L1065fs*

(s) SRSF2_P.P95H
(c) CUX1_P.Q1276*

D

$$
\begin{aligned}
& \text { 응 } \\
& \hline
\end{aligned}
$$

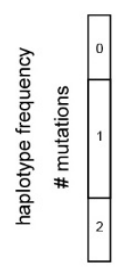

(b) TET2_p.A1224is²

\begin{tabular}{|l|l|l|}
\hline Stem & Progenitor & Differentiated \\
\hline
\end{tabular}

\section{E}
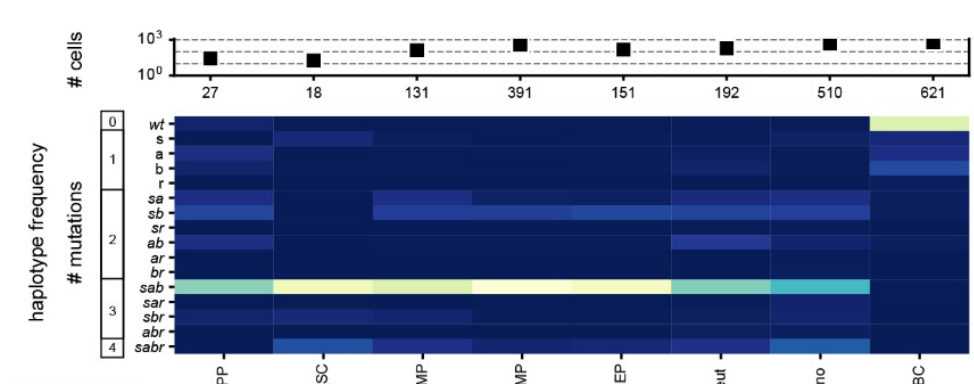

(s) SRSF2_p.P95R

(a) TET2_p.G1218Afs

(b) TET2_p.Y1337*
(r) RUNX1_p.A187T

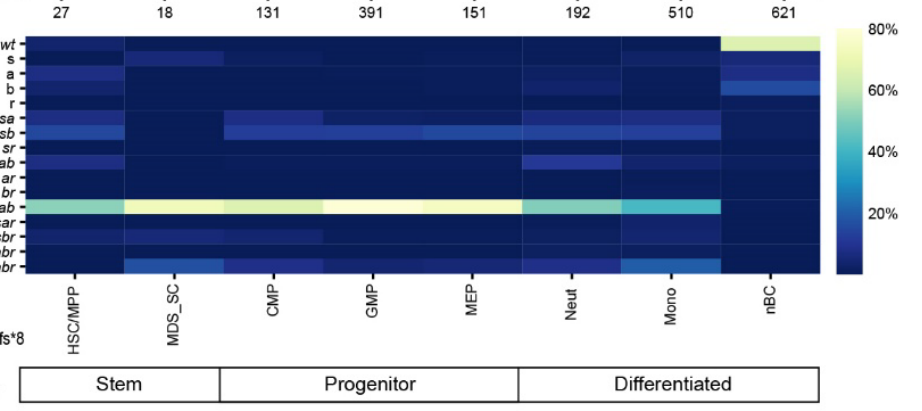

H198302

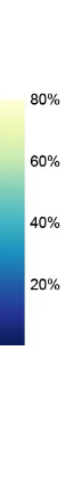

H198303
B

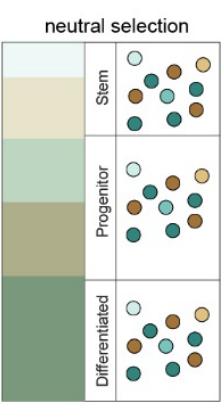

negative clonal selection

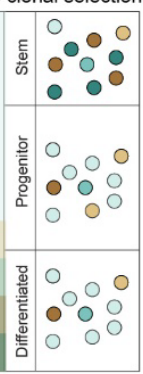

Differentiation to terminal cell types
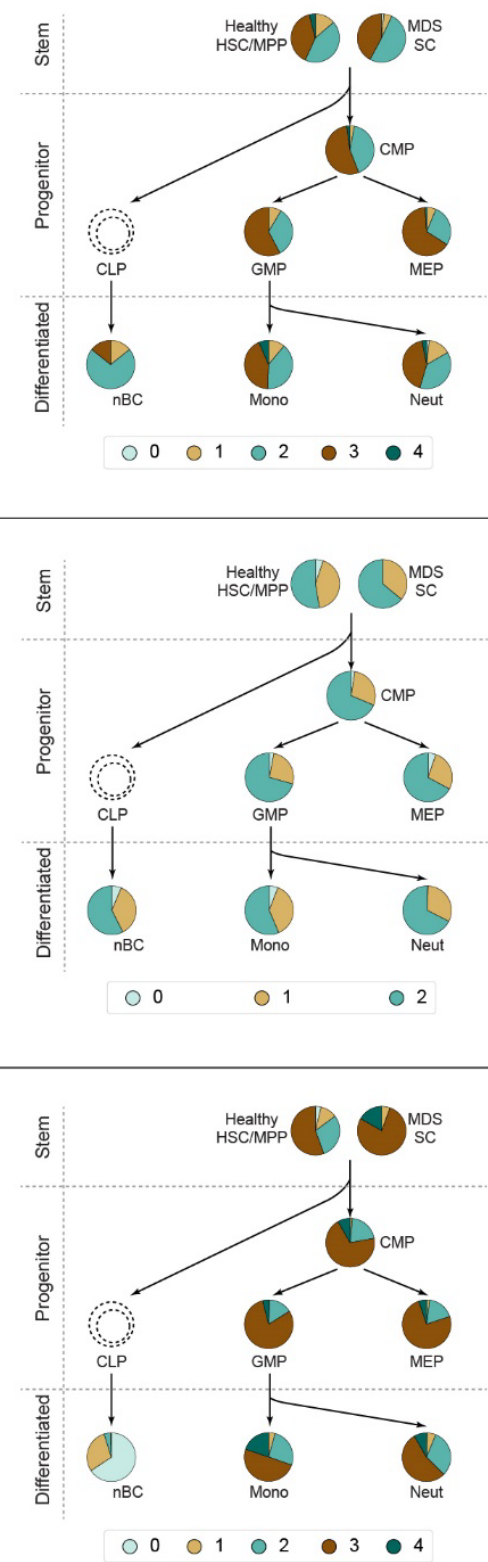

Figure 2 